\title{
Torsion of a rudimentary horn pregnancy_an unusual case presentation
}

\author{
Melissa Blyth • Anthony Griffiths • Richard Penketh
}

Received: 23 May 2012 / Accepted: 28 June 2012 / Published online: 26 July 2012

(C) Springer-Verlag 2012

\section{Introduction}

Aplasia of the mullerian ducts during foetal life can result in the formation of a unicornuate uterus. This can be associated with varying degrees of a rudimentary horn. Of the female population, $5.5 \%$ is thought to have a uterine abnormality, but only $0.1 \%$ has this specific formation [1].

Unicornuate uteri with rudimentary horns are susceptible to many gynaecological and obstetric complications, such as infertility, endometriosis, urinary tract anomalies and premature delivery [2]. They can also, although seldom, undergo horn torsion [3].

Development of a pregnancy in a rudimentary horn is rare occurring in only $1 / 76,000-150,000$ cases [4]. It is made possible when migration of the spermatozoa occurs through the abdominal cavity [5]. Pregnancy carries with it the risk of horn rupture, which can be fatal causing major haemorrhage. Rupture occurs in $80-90 \%$ of cases at midtrimester [6]. When a pregnancy in a rudimentary horn is diagnosed, excision of the horn is often necessary to prevent this, often through a laparotomy and, only more recently, through laparoscopy [5]. Formation of a large uterine horn capable of sustaining a pregnancy is particularly uncommon. The neonatal survival in rudimentary horn pregnancies is only $2 \%[6]$.

Here, we present a case of a patient who developed a pregnancy in a large rudimentary horn with the musculature capable of maintaining a viable pregnancy, but which had also undergone torsion, and who later required laparoscopic management for removal of products of conception. To the best of

M. Blyth $(\bowtie) \cdot$ A. Griffiths $\cdot$ R. Penketh

Department of Obstetrics and Gynaecology,

University Hospital of Wales,

Heath Park, Cardiff, UK

e-mail: Melissa.Blyth@wales.nhs.uk our knowledge, this is the first case report of the management of a torted rudimentary horn pregnancy in the literature.

\section{Case presentation}

A 26-year-old patient presented to the early pregnancy assessment unit with light vaginal bleeding and a positive pregnancy test. Transvaginal scanning revealed a single live foetus of 8 weeks gestation with a normal uterine appearance surrounding the pregnancy. On closer inspection, this large uterine body did not appear to be in continuity with the uterine cervix. An initial diagnosis of pregnancy in a rudimentary horn was suggested.

Additional imaging in the form of an MRI was arranged. This report suggested the presence of a large rudimentary horn that was torted upon its blood supply (Fig. 1).

Management options were discussed with the patient, including methotrexate, surgery or conservative treatment. The patient was keen to maintain her pregnancy if viable but amenable to an operative laparoscopy to assess the anatomy of the horn and correct the uterine torsion.

At laparoscopy, a large uterine horn was found filling the pelvis, connected to a very small vestigial uterine body by a connective tissue bridge. It was this vestigial body that was in continuity with the cervix. The large horn, containing the pregnancy, was torted through a defect in the broad ligament, which with intra-operative Doppler was found to already be occluding its blood supply from the left pelvic side wall. The horn was partially untwisted, and intraoperative scanning confirmed a live foetus at the end of the procedure. Due to patients desire to maintain the pregnancy, laparoscopic excision of the horn was not performed.

Repeat ultrasound scan 1 week later unfortunately revealed a missed miscarriage. Management options of removal of products of conception now had to be discussed with the 


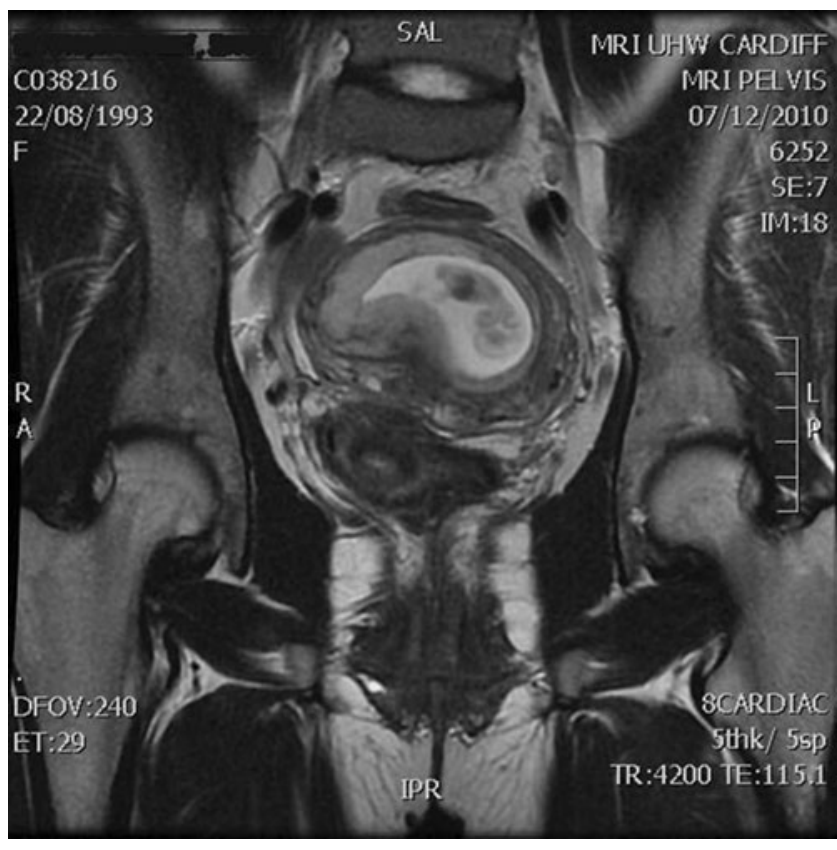

Fig. 1 MRI image of the rudimentary horn pregnancy

patient. Since there was no significant cervical connection, medical and surgical evacuation of retained products would not be possible. The patient was again offered removal of the uterine horn, and options for surrogacy were explained. In the end, after discussion with numerous colleagues and full consultation with the patient, a laparoscopic hysterotomy was performed, and horn was fully untorted.

Patient suffered no postoperative complications and was discharged with a follow-up appointment. She was clearly warned about the risks of future pregnancies. To date, this patient has not returned for further review.

\section{Discussion}

Pregnancy in a rudimentary horn is unusual, but this case is, particularly so as the lateral rudimentary horn, was large and well developed, consistent with normal uterine dimensions. When a uterine horn contains relatively abundant uterine muscle, it can undergo hypertrophy and tolerate dilatation and so may be capable of maintaining a viable pregnancy [4].

Risks of pregnancy in a rudimentary horn, such as uterine rupture, bleeding and undiagnosed premature labour, are well known, but the relative risk is difficult to quantify. It is likely that the incidence of rupture is inversely proportional to the size of the uterine horn, and that diminished muscle mass plays a part in second trimester miscarriage and premature delivery [2]. It was deemed that, due to the large size of the uterine body, the rupture rate was low, and after counselling, continuation of pregnancy was not thought to be contraindicated.
Torsion of a rudimentary horn in pregnancy is extremely rare. To our knowledge, only one case has been reported in the literature, and this occurred at 22 weeks gestation when the pregnancy was in the main uterus [3]. Given this fact, we believe that this is the first case of torsion in a uterine horn pregnancy and, therefore, difficult to manage in an evidencebased manner as there are no previous publications to give guidance.

The management of this case was compounded by the desire of the patient to retain her fertility. Removal of the horn would have left only a very small supracervical unicornuate horn that could not maintain a pregnancy. As this patient refused to entertain the notion of surrogacy and did not consent to removal of the horn, a hysterotomy was felt to be the best option for treatment.

In the previous case report of rudimentary horn torsion, a laparotomy was performed to remove the horn [3]. Laparoscopic resection of a pregnant rudimentary horn is still a very rare event [5]. This case shows how laparoscopy can be used to remove retained products of conception and to untwist horn torsion.

\section{Conclusion}

This case of a torted rudimentary horn pregnancy shows the complexities of dealing with rare uterine abnormalities. In such situations, when unable to manage a case scientifically, we feel that it is important to consult various colleagues and crucial to carefully counsel the patient before any management decision is made.

Conflict of interest None.

\section{References}

1. Chan YY, Jayaprakasan K, Zamora J, Thornton JG, Raine-Fenning N, Coomarasamy A (2011) The prevalence of congenital uterine anomalies in unselected and high risk populations: a systematic review. Hum Reprod Update 17(6):761-771. doi:10.1093/humupd/dmr028

2. Donderwinkel P, Dorr J, Willemsen W (1992) The unicornuate uterus: clinical implications. Eur J Obstet Gynaecol Reprod Biol 47:135-139

3. Wang B, Zhou J, Jin H (2011) Torsion of a rudimentary uterine horn at 22 weeks gestation. J Obstet Gynaecol Res 37(7):919-920. doi:10.1111/j.1447-0756.2010.01435.x

4. Shin J, Kim H (2005) Case of live birth in a non-communicating rudimentary horn pregnancy. J Obstet Gynaecol Res 31(4):329-331

5. Dicker D, Nitke S, Shoenfeld A, Fish B, Mrizner I, Ben-Rafael Z (1998) Laparoscopic management of rudimentary horn pregnancy. Hum Reprod 13(9):2643-2644

6. Daskalaskis G, Pilalis A, Lykeridou K, Antsaklis A (2002) Rupture of noncommunicating rudimentary uterine horn pregnancy. Am Coll Obstet Gynaecol 100(5):1108-1110 